\title{
Nailfold digital capillaroscopic findings in patients with diffuse and limited cutaneous systemic sclerosis
}

\author{
Saeedeh Shenavandeh ${ }^{1}$, Mahyar Yousefipour Haghighi' ${ }^{1}$, Mohammad Ali Nazarinia ${ }^{2}$ \\ ${ }^{1}$ Division of Rheumatology, Department of Internal Medicine, Shiraz University of Medical Science, Iran \\ ${ }^{2}$ Shiraz Geriatric Research Center, Shiraz University of Medical Sciences, Iran
}

\begin{abstract}
Background: Systemic sclerosis (SSc) is a chronic disease with microvascular damage. Nailfold capillaroscopy is a non-invasive method used for evaluating capillaries in SSc. Its findings could be related to the internal organ involvement and SSc course. In this study, we aimed to determine the association of the capillaroscopic patterns of nailfold capillaries with the disease subtypes of SSc, disease duration, and clinical manifestations.

Material and methods: Seventy patients with SSc (15 cases with diffuse cutaneous SSc [DcSSc] and 55 patients with limited SSc [LCSSc]) were studied. The patients were classified into early and intermediate/late DcSSc and LCSSc regarding their disease duration. The capillaroscopy findings were classified into normal, 'early', 'active' and 'late' scleroderma patterns, and 'non-specific' changes. The association of the nailfold capillaroscopy changes and their components with clinical manifestations was also studied.

Results: We studied 15 DcSSC and 55 LCSSc patients. No association was found between the patterns of capillaroscopic changes and these subtypes. There were 8 early DcSSc, 7 intermediate/late DcSSc, 34 early LCSSc, and 21 intermediate/late LcSSc patients. In patients with LcSSc, the 'early' scleroderma pattern of capillaroscopy was associated with early disease based on duration. We found a direct association between some capillary components and some clinical findings. Also, some capillaroscopic components had an inverse association with some clinical manifestations. Conclusions: We found no association between the patterns of capillaroscopy and SSc subtypes; early scleroderma pattern of capillaroscopy was significantly associated with early LcSSc, compatible with the slower course of the disease in LCSSc. Subtle changes, capillary elongation, and capillary tortuosity had an inverse association with clinical manifestations and might be considered as good prognostic factors.
\end{abstract}

Key words: systemic sclerosis, nailfold capillaroscopy, clinical manifestations.

\section{Introduction}

Systemic sclerosis (SSc) is a chronic and often progressive connective tissue disease with unknown etiology. There are two subsets of the disease involving the skin: DcSSc (diffuse cutaneous SSc) is the rapidly progressive type; and limited cutaneous SSc (LCSSc), which is generally identified by Raynaud's phenomenon before other manifestations, progresses slowly [1]. The pathophysiology of the disease is ischemia and fibrosis due to loss of peripheral capillaries, lack of capillary repair, and new revascularization [2].

Nailfold capillaroscopy is a non-invasive, cheap and useful method used to detect some connective tissue diseases, and it differentiates the primary Raynaud's phenomenon from the secondary one [3]. It can be used for early diagnosis of SSc and observation of development of microangiopathy in SSc [4]. In patients with scleroderma, the capillary changes usually include di-

Address for correspondence:

Saeedeh Shenavandeh, Division of Rheumatology, Department of Internal Medicine, Shiraz University of Medical Science, $71345-1414$ Shiraz, Iran, e-mail: shenavandehs@gmail.com

Submitted: 24.11.2016, Accepted: 7.02.2017 
lated and giant capillaries, hemorrhages, disorganized vascular arrays, ramified/bushy capillaries, and capillary losses [3]. The overall changes are classified into 'early', 'active', and 'late' scleroderma patterns [5]. Nailfold capillaroscopy (NFC) is recommended as part of screening and monitoring the process of SSc [6].

In this study, we aimed to study nailfold capillaries in patients with DcSSc and LCSSc. Both groups were divided into early and intermediate/late subgroups based on duration ( $<3$ and $\geq 3$ years in DcSSc; $<5$ and $\geq 5$ years in LCSSc) $[7,8]$. Additionally, the association of the clinical manifestations and skin score with the capillaroscopy patterns and components was studied.

\section{Material and methods}

This article was agreed by Bioethic Committee of Shiraz University of Medical Science. We studied all the patients with scleroderma referred to Hafez Hospital Scleroderma Clinic or those who were admitted in the Hafez Rheumatology Ward from 20th of April 2012 to 20th of April 2013. Seventy out of 73 patients fulfilled the 1980 American College of Rheumatology (ACR) criteria for systemic sclerosis and new (2013) ACR/EULAR SSc diagnostic criteria [9]. The patients should have had at least 6 evaluable nailfolds to be looked through for the microscopic procedure. In all patients, 8 nailfolds including all fingers of the hands except for the two thumbs were evaluated [10]. Patients with overlap syndrome, diabetes mellitus, and smokers were excluded from the study. Also, those with limitations in capillary evaluation such as very thick and ulcerated fingers in all fingers were excluded from the study.

After having a declaration of consent form signed by the patients, all clinical manifestations of the patients, any organ involvement, and any available para-clinical and laboratory data were recorded. Demographic information, disease specifications, skin score, clinical manifestations, physical examination findings including presence of pitting scars and finger-to-palm (FTP), VAS, para-clinical, and laboratory data were recorded by the rheumatologist in the screen forms. The SSc patients were assessed for sex, age, disease duration (date of first Raynaud's and non-Raynaud's symptoms), serological characteristics [(anti-Scl-70, anti-centromere antibodies (ACA) or antinuclear antibody (ANA)] and skin scoring according to the definition by LeRoy et al. [10] by MRSS (Modified Rodnan Skin Score) scoring, pulmonary involvement according to high resolution computed tomography scan (HRCT) at the first screening or after detecting low DLCO $<80 \%$ or low $\mathrm{FEV}_{1}<75 \%$ in the pulmonary function test (PFT), vascular involvement by cutaneous ulcerations, and pulmonary artery hypertension (PAH) by echocardiography in all scleroderma patients as a basic evaluation of the clinic.
According to the cross-sectional disease duration, skin score, and skin involvement extent, the patients were divided into 4 subtypes/subgroups as early diffuse cutaneous SSc (DcSSc) (<3 years) and intermediate/late ( $>3$ years) DcSSc and early limited cutaneous SSc (LCSSc) ( $<5$ years) and intermediate/late LcSSc ( $>5$ years) $[7,8]$.

Nailfold capillaroscopy was done by the rheumatologist involved in the study using the Hafez Hospital stereomicroscope Euromex ST. 1740, made in Holland, at $\times 250$ power and the video camera Cmex D.C.5000 (5 megapixels). Immersion oil was applied on the nailfold bed to improve the resolution. Eight fingers of the two hands excluding the thumbs were assessed. Fingers with thick nailfolds and ulcerated ones were not studied. We also took at least one image of the middle of the nailfold per finger. The data including distribution (disturbed or normal), shape of capillaries (normal hairpin, subtle change including tortuosity and crossing, anomalies including meandering, ectasia, ramification/bushy/ arborization/branching (neoangiogenesis) and bizarre, the largest diameter of arterial or venous side [dilated loops were defined as irregular or homogeneous increase of capillary diameter $\geq 20$ and $<50 \mu \mathrm{m}$ and giant capillaries (a capillary with a homogeneously enlarged loop with a diameter $\geq 50 \mu \mathrm{m}$ )], capillary length (normal or elongated: $\geq 300 \mu \mathrm{m}$ ), mean capillary density (capillary loss was defined as reduction of the normal number of capillaries below 7 per linear millimeter), avascular area (intercapillary distance $>500 \mu \mathrm{m}$ ): present or absent, microhemorrhages (presence of one or more dark red masses characterized by hemosiderin deposits due to capillary injury or thrombosis): present or absent and neoangiogenesis or ramified capillaries (branching, bushy, interconnected capillaries, originating from a single capillary): present or absent were recorded in a form. The whole findings were defined as normal; specific changes for 'early', 'active', and 'late' scleroderma pattern; or 'non-specific' changes. 'Early' scleroderma pattern was defined as few enlarged/giant capillaries, few capillary hemorrhages, relatively well-preserved capillary distribution, no evident loss of capillaries, 'active' as frequent giant capillaries, frequent capillary hemorrhages, moderate loss of capillaries, mild disorganization of the capillary architecture and absent or mild ramified capillaries and 'late' as irregular enlargement of the capillaries, few or absent giant capillaries and hemorrhages, severe loss of capillaries with extensive avascular areas, disorganization of the normal capillary array, and ramified/bushy capillaries [5]. The 'non-specific' changes were divided into minor and major changes. Presence of subtle capillary changes including tortuosity, crossing, and mixed patterns was also recorded. The overall capillaroscopic patterns were defined as normal 
(6-8 capillaries/mm, capillary length between 200-500 $\mu \mathrm{m}$, hair pin shaped loops arranged in parallel rows, absence of hemorrhages), minor abnormalities (6-8 capillaries/mm, less than 50\% tortuous loops, arranged in parallel rows, with no hemorrhages), major abnormalities (normal or decreased capillary density, more than 50\% tortuous, enlarged loops, disarranged, with hemorrhages), and typical scleroderma patterns (decreased number of capillaries, tortuous, branched, bushy, enlarged, giant, disarranged, with hemorrhages). Minor and major changes without a specific scleroderma pattern were defined as 'non-specific' changes [11]. Finally, the relationship between the disease subtypes and scleroderma patterns was studied.

The data were analyzed using the chi-squared test in SPSS 20 for Windows software. Results of quantitative data are shown as mean \pm standard deviation. A $p$-value $<0.05$ was considered statistically significant.

\section{Results}

Among all 73 primary cases, 3 patients did not fulfill the inclusion criteria and 70 patients were enrolled in the study.

The demographic data, disease specifications, and disease subgroups' frequency and means are presented in Table I.

Capillaroscopy patterns and their components are shown in Table II. Two cases with 'non-specific' pattern of capillaroscopy were detected in the LCSSc group in which one had major capillaroscopic changes and the other minor ones.

Skin score and clinical manifestations' frequency and means are presented in Table III. The association between the SSc subtype/subgroups with scleroderma patterns of capillaroscopy was evaluated. There was no association between the SSc subtypes (DcSSc and LCSSc) and scleroderma patterns of capillaroscopy.

In dividing each of the DcSSC and LCSSc disease subtypes into early and intermediate/late subdivisions, early LCSSc was associated with 'early' capillaroscopy scleroderma pattern in contrast to the 3 other subgroups. The data are shown in Table IV.

The associations of the skin score, clinical manifestations, and para-clinical data with scleroderma patterns of capillaroscopy in different SSc subtypes (DcSSc and LCSSc) and subgroups (early and late SSc) were evaluated and the positive findings are presented in Table $\mathrm{V}$.

In analysis of the associations of the skin score, clinical manifestation, VAS, para-clinical data, and laboratory tests with scleroderma patterns of capillaroscopy in different SSc subtypes (DcSSc and LcSSc) and subgroups (early and late SSc) 'late' and 'active' scleroderma pattern of capillaroscopy were more prevalent in patients with skin telangiectasia (in all patients, early SSc, and LcSSc subtypes/subgroups), pitting scars (in all patients and LCSSc subtype), and pulmonary rales (in all patients) compared to those with 'early' scleroderma pattern. Skin score was not significantly associated with the scleroderma patterns of capillaroscopy.

$\mathrm{FEV}_{1}<70 \%$ had no significant association with the scleroderma patterns of capillaroscopy when studied in all patients, but when analyzed in the SSc subgroups/ subtypes (Early SSc\& Late SSc subgroups and LCSSc and DcSSc subtypes) separately, it was significantly more frequent in patients with 'late' scleroderma pattern of capillaroscopy than 'active' and 'early' scleroderma patterns sequentially only in the early SSc subgroup and LCSSC subtype. However, such an association was found neither in the late SSc subgroup nor in the DcSSc subtype.

Limitation of the finger-to-palm (FTP) range of motion of each finger, when studied in all patients, was significantly associated with 'late' scleroderma pattern of capillaroscopy.

Skin score, clinical manifestation, VAS, paraclinical, and laboratory data were again analyzed in association with the scleroderma patterns of capillaroscopy in the 4 different SSc subtypes/subgroups.

Some of the capillary changes had direct associations with clinical and para-clinical findings. For example,

Table I. Demographic data, disease information, and disease subgroups' frequency

\begin{tabular}{|c|c|c|c|c|c|c|c|c|}
\hline \multicolumn{2}{|l|}{ Variable } & $\begin{array}{l}\text { All patients } \\
\quad(n=70)\end{array}$ & $\begin{array}{l}\text { DcSSc } \\
(n=15)\end{array}$ & $\begin{array}{l}\text { LcSSc } \\
(n=55)\end{array}$ & $\begin{array}{l}\text { Early DcSSc } \\
\qquad(n=8)\end{array}$ & $\begin{array}{l}\text { Late DcSSc } \\
\qquad(n=7)\end{array}$ & $\begin{array}{l}\text { Early LcSSc } \\
\qquad(n=34)\end{array}$ & $\begin{array}{l}\text { Late LcSSc } \\
\qquad(n=21)\end{array}$ \\
\hline \multirow[t]{2}{*}{ Gender } & Male no. (\%) & 11 (15.7\%) & $4(26.7 \%)$ & 7 (12.7\%) & $1(12.5 \%)$ & 3 (42.9\%) & $4(11.8 \%)$ & $3(14.3 \%)$ \\
\hline & Female no. (\%) & $59(84.3 \%)$ & $11(73.3 \%)$ & $48(87.3 \%)$ & $7(87.5 \%)$ & $4(57.1 \%)$ & $30(88.2 \%)$ & $18(85.7 \%)$ \\
\hline \multicolumn{2}{|c|}{ Age (years old) mean \pm SD } & $41.5 \pm 11$ & $40 \pm 13.5$ & $41.9 \pm 10.3$ & $32.5 \pm 5$ & 48.6 & $42.6 \pm 9.6$ & $40.6 \pm 11.5$ \\
\hline \multicolumn{2}{|c|}{$\begin{array}{l}\text { Disease } \\
\text { Duration (years) mean } \pm S D\end{array}$} & $4.8 \pm 6$ & $3.5 \pm 5$ & $5.1 \pm 5.8$ & $0.8 \pm 0.3$ & $6.6 \pm 6$ & $1.9 \pm 1.2$ & $10.3 \pm 6.5$ \\
\hline \multicolumn{2}{|c|}{$\begin{array}{l}\text { RP } \\
\text { Duration (years) mean } \pm \text { SD }\end{array}$} & $6 \pm 7$ & $3.4 \pm 5$ & $6.7 \pm 7.4$ & $1.2 \pm 0.9$ & $5.8 \pm 6.6$ & $3.1 \pm 4.6$ & $12.6 \pm 7.3$ \\
\hline
\end{tabular}

$R P$ - Raynaud's phenomenon; SD - standard deviation 
Table II. Capillaroscopic scleroderma patterns and its components' frequency

\begin{tabular}{|c|c|c|c|c|c|c|c|c|}
\hline \multicolumn{2}{|l|}{ Variable } & $\begin{array}{c}\text { All patients } \\
(n=70) \\
\text { no. }(\%)\end{array}$ & $\begin{array}{c}\text { DcSSc } \\
(n=15) \\
\text { no. }(\%)\end{array}$ & $\begin{array}{c}\text { LcSSc } \\
(n=55) \\
\text { no. (\%) }\end{array}$ & $\begin{array}{c}\text { Early DcSSc } \\
(n=8) \\
\text { no. }(\%)\end{array}$ & $\begin{array}{c}\text { Late DcSSc } \\
(n=7) \\
\text { no. }(\%)\end{array}$ & $\begin{array}{c}\text { Early LcSSc } \\
\quad(n=34) \\
\text { no. }(\%)\end{array}$ & $\begin{array}{c}\text { Late LcSSc } \\
(n=21) \\
\text { no. }(\%)\end{array}$ \\
\hline \multicolumn{2}{|c|}{ Scleroderma pattern } & $68(97.1 \%)$ & 15 (100\%) & $53(96.4 \%)$ & $8(100 \%)$ & $7(100 \%)$ & $33(97.1 \%)$ & $20(95.2 \%)$ \\
\hline \multirow[t]{4}{*}{$\begin{array}{l}\text { Capillary } \\
\text { pattern }\end{array}$} & $\begin{array}{l}\text { "early" scleroderma } \\
\text { pattern }\end{array}$ & $16(22.9 \%)$ & $1(6.7 \%)$ & $15(27.3 \%)$ & $1(12.5 \%)$ & $0(0 \%)$ & $14(41.2 \%)$ & $1(4.8 \%)$ \\
\hline & $\begin{array}{l}\text { "active" scleroderma } \\
\text { pattern }\end{array}$ & $34(48.6 \%)$ & $8(53.3 \%)$ & $26(47.3 \%)$ & $5(62.5 \%)$ & $3(42.9 \%)$ & $16(47.15 \%)$ & $10(47.6 \%)$ \\
\hline & $\begin{array}{l}\text { "late" scleroderma } \\
\text { pattern }\end{array}$ & $18(25.7 \%)$ & $6(40 \%)$ & $12(21.8 \%)$ & $2(25 \%)$ & $4(57.1 \%)$ & $4(11.8 \%)$ & $8(38.1 \%)$ \\
\hline & "nonspecific" pattern & $2(2.9 \%)$ & $0(0 \%)$ & $2(3.6 \%)$ & $0(0 \%)$ & $0(0 \%)$ & $0(0 \%)$ & $2(9.5 \%)$ \\
\hline \multicolumn{2}{|c|}{ Abnormal capillary distribution } & $55(78.6 \%)$ & $14(93.3 \%)$ & $41(74.5 \%)$ & $8(100 \%)$ & $6(85.7 \%)$ & $21(61.8 \%)$ & $20(95.2 \%)$ \\
\hline \multicolumn{2}{|c|}{ Subtle change } & $15(21.4 \%)$ & $0(0 \%)$ & $15(27.3 \%)$ & $0(0 \%)$ & $0(0 \%)$ & $11(32.4 \%)$ & $4(19 \%)$ \\
\hline \multicolumn{2}{|c|}{ tortuosity } & $2(11.4 \%)$ & $0(0 \%)$ & $8(14.5 \%)$ & $0(0 \%)$ & $0(0 \%)$ & $6(17.6 \%)$ & $2(9.5 \%)$ \\
\hline \multicolumn{2}{|l|}{ crossing } & $2(12.9 \%)$ & $0(0 \%)$ & $9(16.4 \%)$ & $0(0 \%)$ & $0(0 \%)$ & $7(20.6 \%)$ & $2(9.5 \%)$ \\
\hline \multicolumn{2}{|l|}{ mixed } & $0(0 \%)$ & $0(0 \%)$ & $0(0 \%)$ & $0(0 \%)$ & $0(0 \%)$ & $0(0 \%)$ & $0(0 \%)$ \\
\hline \multicolumn{2}{|c|}{ Capillary anomalies } & 65 (92.9\%) & $12(80 \%)$ & $53(96.4 \%)$ & $6(75 \%)$ & $6(85.7 \%)$ & 33 (97.1\%) & $20(95.2 \%)$ \\
\hline \multicolumn{2}{|c|}{ widening } & $6(8.6 \%)$ & $2(13.3 \%)$ & $4(7.3 \%)$ & $1(12.5 \%)$ & $1(14.3 \%)$ & $3(8.8 \%)$ & $1(4.8 \%)$ \\
\hline \multicolumn{2}{|c|}{ meandering } & $2(2.9 \%)$ & $0(0 \%)$ & $2(3.6 \%)$ & $0(0 \%)$ & $0(0 \%)$ & $1(2.9 \%)$ & $1(4.8 \%)$ \\
\hline \multicolumn{2}{|l|}{ dilation } & 65 (92.9\%) & $13(86.7 \%)$ & $52(94.5 \%)$ & $7(87.5 \%)$ & $6(85.7 \%)$ & $34(100 \%)$ & $18(85.7 \%)$ \\
\hline \multicolumn{2}{|c|}{ neoangiogenesis } & $26(37.1 \%)$ & $9(60 \%)$ & 17 (30.9\%) & $4(50 \%)$ & $5(71.4 \%)$ & $8(23.5 \%)$ & $9(42.9 \%)$ \\
\hline \multicolumn{2}{|l|}{ bizarre } & $6(8.6 \%)$ & $0(0 \%)$ & $6(10.9 \%)$ & $0(0 \%)$ & $0(0 \%)$ & $3(8.8 \%)$ & $3(14.3 \%)$ \\
\hline \multicolumn{2}{|c|}{ Giant loop (> $0.05 \mathrm{~mm})$} & $44(62.9 \%)$ & $7(46.7 \%)$ & $37(67.3 \%)$ & $5(62.5 \%)$ & $2(28.6 \%)$ & $26(76.5 \%)$ & $11(52.4 \%)$ \\
\hline \multicolumn{2}{|c|}{ Reduced capillary density $<7 / \mathrm{mm}$} & $49(70 \%)$ & $14(93.3 \%)$ & $35(63.6 \%)$ & $7(87.5 \%)$ & $7(100 \%)$ & $21(61.8 \%)$ & $13(60.7 \%)$ \\
\hline \multicolumn{2}{|c|}{ Avascular area } & $27(38.6 \%)$ & $7(46.7 \%)$ & $20(36.4 \%)$ & $3(37.5 \%)$ & $4(57.1 \%)$ & $11(32.4 \%)$ & $9(42.9 \%)$ \\
\hline \multicolumn{2}{|c|}{ Hemorrhage } & $46(65.7 \%)$ & $7(46.7 \%)$ & $39(70.9 \%)$ & $5(62.5 \%)$ & $2(28.6 \%)$ & $26(76.5 \%)$ & $13(61.9 \%)$ \\
\hline \multicolumn{2}{|c|}{ Elongated capillary } & $10(14.3 \%)$ & $1(6.7 \%)$ & $9(16.4 \%)$ & $1(12.5 \%)$ & $0(0 \%)$ & $7(20.6 \%)$ & $2(9.5 \%)$ \\
\hline \multicolumn{2}{|c|}{ Abnormal blood flow } & $53(85 \%)$ & $7(58.3 \%)$ & $46(92 \%)$ & $2(28.6 \%)$ & $4(60 \%)$ & $3(9.1 \%)$ & $1(5.9 \%)$ \\
\hline
\end{tabular}

LCSSC - limited cutaneous systemic sclerosis; DcSSC - diffuse cutaneous systemic sclerosis

presence of neoangiogenesis $(p=0.000)$, reduced capillary density $(p=0.002)$, avascular area $(p=0.040)$, and hemorrhages $(p=0.014)$ in nailfold capillaroscopy were directly associated with the presence of limitation of FTP range of motion of the fingers. Presence of neoangiogenesis ( $p=0.003)$ was directly associated with pitting scars, too. Presence of avascular area $(p=0.049)$ had a direct association with gastrointestinal (GI) problems including any of the followings: dysphagia, heart burn, difficulty swallowing, the feeling of being full, vomiting, diarrhea, and constipation. Also, the presence of giant loops $(p=0.033)$ was directly associated with dysphagia. Abnormal blood flow detection in capillaroscopy ( $p=0.003)$ was also directly associated with positive CRP, when they were studied in all patients.

Similar results were found in the early and late SSC separately. Presence of neoangiogenesis $(p=0.000)$ was directly associated with limitation of FTP range of motion of the fingers when studied in early SSc. Abnormal blood flow $(p=0.010)$ revealed a direct relationship with high skin score $>15$. Abnormal blood flow $(p=0.037)$, neoangiogenesis ( $p=0.046)$, and reduced capillary density ( $p$ $=0.026$ ) were directly related to limitation of FTP range of motion of fingers, too. Abnormal blood flow was also directly associated with higher means of the skin score ( $p=0.040)$ and higher means of VAS of Raynaud's phenomenon $(p=0.022)$, all when studied in late SSc.

Some capillary changes had an inverse association with some clinical findings, such as presence of subtle changes, which had an inverse association with GI problems ( $p=0.039)$ and limitation of FTP range of motion of fingers $(p=0.012)$. Capillary elongation had an inverse association with pitting scars ( $p=0.001)$, too. Also, presence of capillary tortuosity showed an inverse association with peripheral vascular problems $(p=0.007)$ (Fig. 1). 
Table III. The skin score and clinical manifestation frequency in all patients and two subtypes of SSc (DcSSc and LCSSC) separately

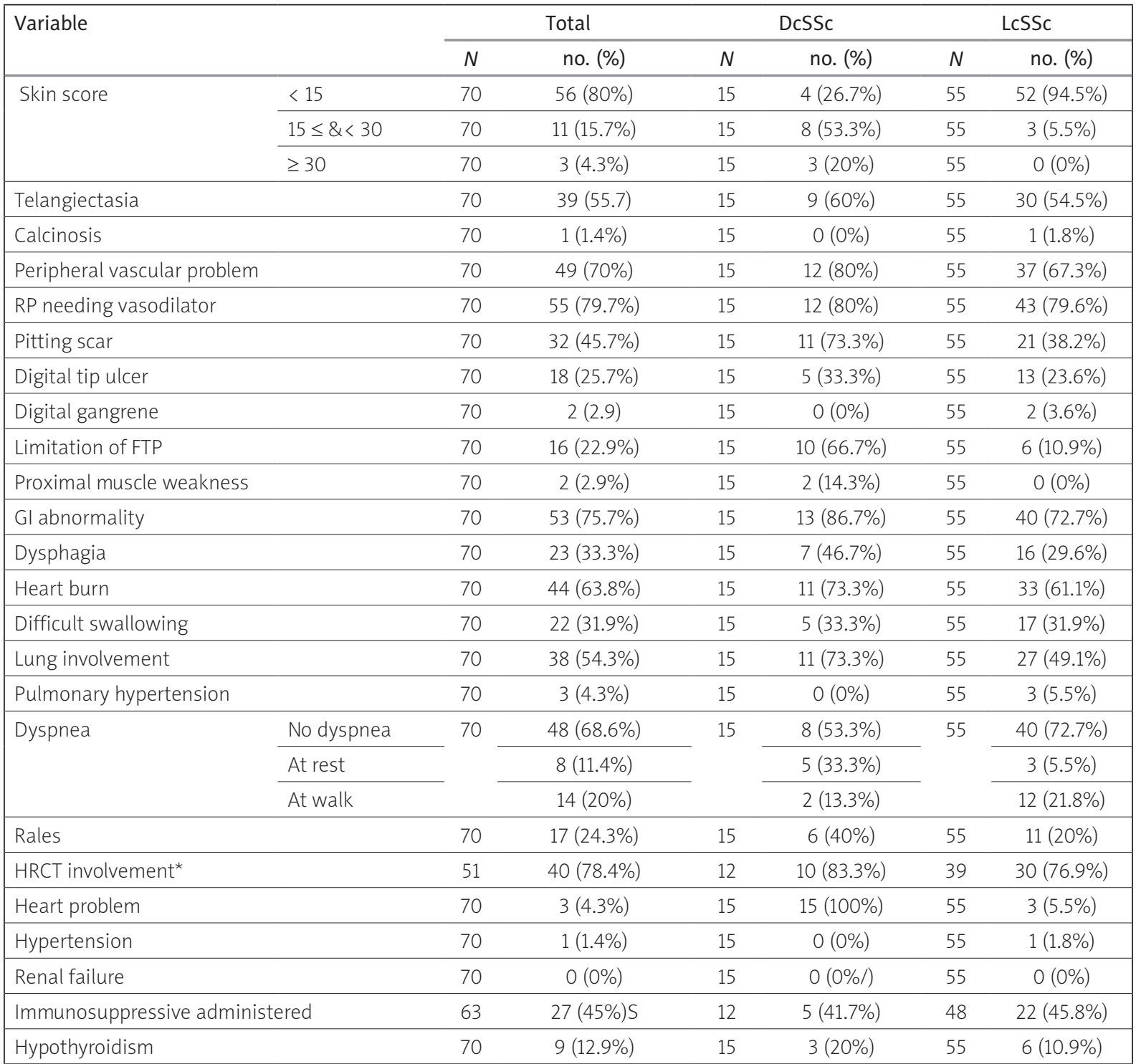

RA - Raynaud's phenomenon; FTP - finger-to-palm; GI - gastrointestinal; LCSSC - limited cutaneous systemic sclerosis; DcSSC-diffuse cutaneous systemic sclerosis

*Including: ground glass opacities, reticular patterns, or honeycombing

Table IV. The association between the SSc subtypes/subgroups with scleroderma patterns of capillaroscopy

\begin{tabular}{|lcccccc|}
\hline SSc subtype & \multicolumn{3}{c}{ Scleroderma pattern of capillaroscopy } & & $P$ & $N$ \\
\cline { 2 - 5 } & "early" & "active" & "late" & "non specific" & & \\
\hline Early DcSSc & $1(12.5 \%)$ & $5(62.5 \%)$ & $2(25 \%)$ & $0(0 \%)$ & 0.007 & 70 \\
\hline Late DcSSc & $0(0 \%)$ & $3(42.9 \%)$ & $4(57.1 \%)$ & $0(0 \%)$ & & \\
\hline Early LCSSc & $14(41.2 \%)$ & $16(47.1 \%)$ & $4(11.8 \%)$ & $0(0 \%)$ & & \\
\hline Late LCSSc & $1(4.8 \%)$ & $10(47.6 \%)$ & $8(38.1 \%)$ & $2(9.5 \%)$ & & \\
\hline
\end{tabular}

SSC - systemic sclerosis; LCSSC - limited cutaneous systemic sclerosis; DCSSC - diffuse cutaneous systemic sclerosis 
Table V. Detailed analysis of the significant associations between clinical manifestation and para-clinical data and capillaroscopic patterns in 1) all patients, 2) DcSSc and 3) LCSSc besides 4) early and 5) late SSc analyzed separately

\begin{tabular}{|lcccccc|}
\hline \multirow{2}{*}{ Variable } & \multicolumn{3}{c}{ Scleroderma pattern of capillaroscopy } & \multirow{2}{*}{$P$} & \multirow{2}{*}{ Total $N}$. \\
\cline { 2 - 5 } & “early" & “active" & “late” & “non specific" & & \\
\hline Telangiectasia in all SSc cases & $3(18 \%)$ & $23(67.6 \%)$ & $13(72.2 \%)$ & $0(0 \%)$ & 0.001 & 70 \\
\hline Telangiectasia in early SSc & $2(13.3 \%)$ & $12(57.1 \%)$ & $3(50 \%)$ & $0(0 \%)$ & 0.023 & 42 \\
\hline Telangiectasia in LcSSc & $3(20 \%)$ & $18(69.2 \%)$ & $9(75)$ & $0(0 \%)$ & 0.002 & 55 \\
\hline Pitting scar in all SSc cases & $3(18 \%)$ & $16(47 \%)$ & $13(72 \%)$ & $0(0 \%)$ & 0.007 & 70 \\
\hline Pitting scar in LcSSc & $2(9.1 \%)$ & $11(45.5 \%)$ & $8(45.5 \%)$ & $0(0 \%)$ & 0.018 & 55 \\
\hline Limitation of FTP in all SSc cases & $1(6.2 \%)$ & $6(17.6 \%)$ & $9(50 \%)$ & $0(0.0 \%)$ & 0.013 & 70 \\
\hline Limitation of FTP in DcSSc & $1(100 \%)$ & $3(37.5 \%)$ & $6(100 \%)$ & $0(0 \%)$ & 0.025 & 15 \\
\hline Rales in all SSc cases & $0(0.0 \%)$ & $10(29.4 \%)$ & $7(38.9 \%)$ & $0(0.0 \%)$ & 0.020 & 70 \\
\hline Low FEV1 (<70\%) in LCSSc & $0(0.0 \%)$ & $3(50.0 \%)$ & $3(100 \%)$ & $0(0.0 \%)$ & 0.024 & 15 \\
\hline Low FEV1 (< 70\%) in early DcSSc & $0(0.0 \%)$ & $1(25 \%)$ & $2(100 \%)$ & $0(0 \%)$ & 0.048 & 11 \\
\hline
\end{tabular}

SSC - systemic sclerosis; LCSSC - limited cutaneous systemic sclerosis; DCSSC - diffuse cutaneous systemic sclerosis; FTP - finger-to-palm; FEV1 - forced expiratory volume in one second \% of vital capacity
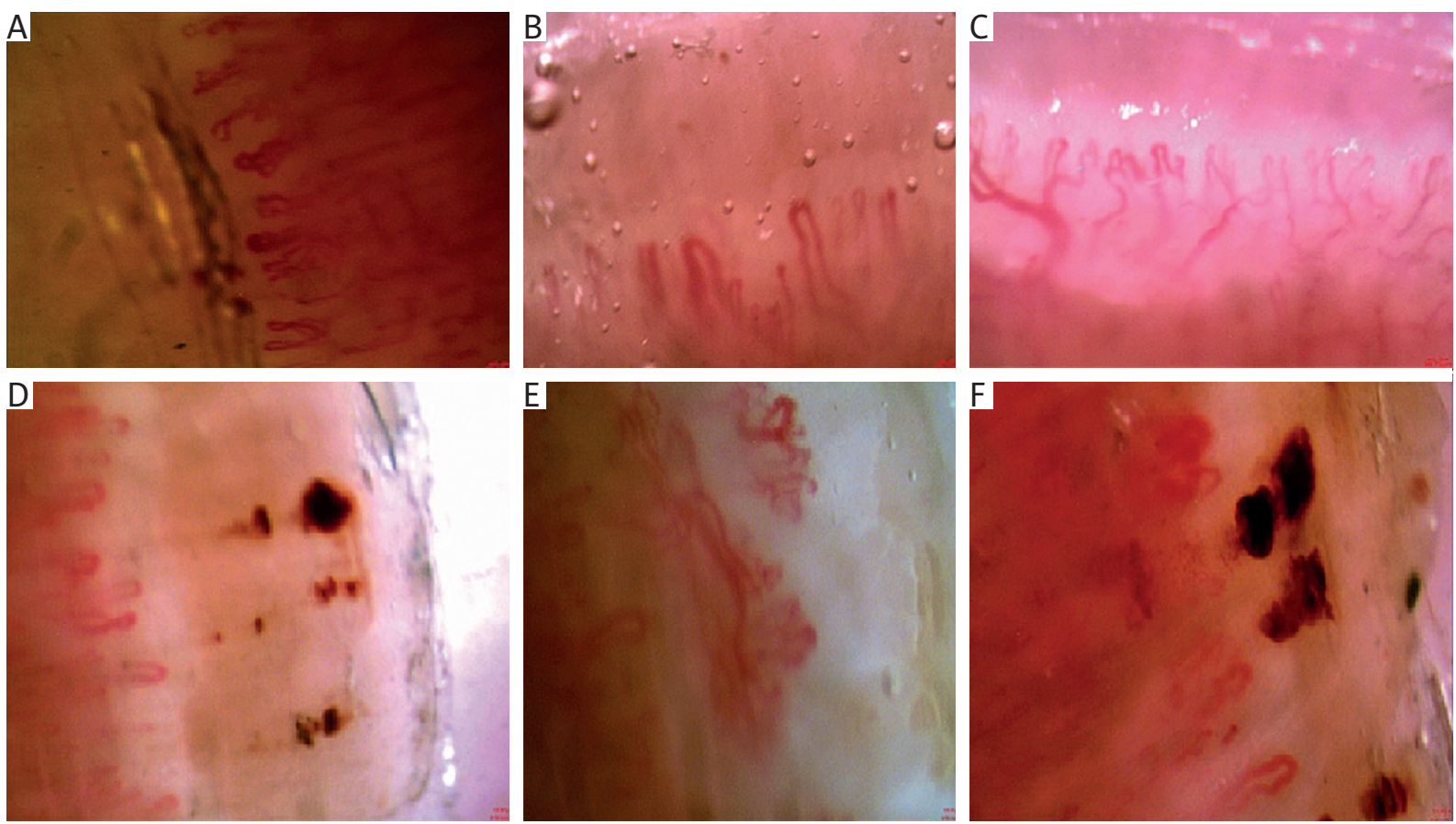

Fig. 1. Tortuous capillaries (A), elongated capillaries (B), scleroderma patterns (C, D, E, F).

\section{Discussion}

This study was designed to evaluate different patterns of capillaries by nailfold capillaroscopy in patients with DcSSc and LcSSc. No significant association was found when we studied them combined with the disease duration dividing the patients into early and intermediate/late. The only significant finding was the association of 'early' scleroderma pattern of capillaroscopy with early $\operatorname{LCSSC}$ ( $<5$ years).
This study revealed that 68 (97.1\%) patients with scleroderma had scleroderma pattern of capillaroscopy and 2 (2.9\%) cases had 'non-specific' changes. Scleroderma patterns of capillaroscopy were not statistically different between patients with DcSSc and LcSSc. In the Ingegnoli et al. [6] study, 2378 (86.35\%) patients had scleroderma patterns of capillaroscopy and 376 (13.65\%) did not. They found 'early' and 'active' scleroderma patterns more prevalent in LcSSc in contrast to DcSSc. Cu- 
tolo et al. [12] found that 'early' and 'active' scleroderma patterns were associated with LCSSc and 'late' scleroderma pattern with DcSSc.

We studied the association of the scleroderma patterns of capillaroscopy with 4 disease subtypes/subgroups based on duration and found that they varied among them. As seen in Table 5, the 'early' scleroderma pattern was significantly more prevalent in early LCSSc than other SSc subtypes/subgroups. This may represent the slow pattern of progression of manifestations of LCSSc. The 'early' scleroderma pattern was also more prevalent statistically in early SSc in contrast to the 'late' scleroderma pattern which was more prevalent in intermediate/late SSc. In this regard, Lambova et al. [7] found the 'early' scleroderma pattern more prevalent in the early phase of SSc ( $<3$ years) and the 'late' pattern more prevalent in the late phase of SSc ( $>3$ years).

Among 55 patients with LCSSc who were divided into early and intermediate/late subgroups, the 'early' scleroderma pattern of capillaroscopy was more prevalent in early LCSSc in contrast to the 'late' scleroderma pattern, which was more prevalent in intermediate/late LCSSc. This difference was not observed in the patients with early DcSSc compared to those with intermediate/ late DcSSc $(n=15)$ (Table V) (i.e. in early years of DcSSc, we can see all scleroderma patterns of capillaroscopy). This result can show the rapid progression of diffuse scleroderma.

Regarding the demographic information, we did not detect any significant association with the scleroderma patterns of capillaroscopy, but Cutolo et al. [12] found higher age, disease duration, and Raynaud's phenomenon duration in 'late' scleroderma pattern compared to 'active' and 'early' patterns. However, the mean SSc duration was not significantly different between different scleroderma patterns according to Ingegnoli et al. [6], as in the present study.

In studying the association of the capillaroscopy patterns of scleroderma ('early', 'active', and 'late' patterns) with the clinical manifestations and paraclinical/laboratory findings, we found that the skin score was not significantly associated with the scleroderma patterns of capillaroscopy. In our study, digital ulcer was not associated with the scleroderma patterns of capillaroscopy. Ingegnoli et al. [6] found digital ulcer more prevalent in the 'late' scleroderma pattern than 'active' and 'early' patterns. In contrast, Lambova et al. [7] found it more prevalent in those with the 'active' scleroderma pattern of capillaroscopy.

FEV $1<70 \%$ had no significant association with the scleroderma patterns when studied in all patients. The patients in our study did not have any renal crisis, so we did not evaluate it in this study. However, in the Ingeg- noli et al. [6] study there was no significant association between the scleroderma patterns and renal crisis.

In our study, pulmonary fibrosis and pulmonary artery hypertension were not associated with the scleroderma patterns of capillaroscopy, but Ingegnoli et al. [6] found it more prevalent in the 'late' scleroderma pattern than 'active' and 'early' patterns. It should be mentioned that we had a few [3 (4.3\%) of all] patients with $\mathrm{PAH}$, so we could not compare it with previous studies, and there is a need for more patients to be evaluated in our study center.

Limitation of FTP range of motion of fingers, in all patients, was significantly associated with 'late' scleroderma pattern of capillaroscopy, which was consistent with the Ingegnoli et al. study [6].

We found no association of anti-Scl 70 antibodies $(n=26)$ or anti-centromere $(n=3)$ antibodies with scleroderma patterns. However, anti-Scl 70 antibodies was significantly more prevalent in those with the 'late' scleroderma pattern of capillaroscopy than 'active' and 'early' ones in the Ingegnoli et al. study [6]. Cutolo et al. [12] found anti-Scl 70 antibodies significantly more prevalent in those with 'late' and 'active' scleroderma patterns of capillaroscopy than the 'early' one, too. Anti-centromere was significantly more prevalent in 'early' and 'active' scleroderma patterns of capillaroscopy than the 'late' one in the Ingegnoli et al. study [6]. Our results might be due to the low number of patients studied for these tests.

Subtle change had an inverse association with both GI problem and limitation of FTP range of motion of fingers. Also, capillary tortuosity had an inverse association with peripheral vascular problem. Neoangiogenesis had a direct association with pitting scars and limitation of FTP range of motion of fingers. Presence of giant loops had a direct association with dysphagia. Avascular area also had a direct association with GI problem and limitation of FTP range of motion of fingers. Presence of micro-hemorrhages was associated with limitation of FTP range of motion of fingers. Presence of elongated capillaries had an inverse association with pitting scars.

Compared to the literature, we found that presence of capillary elongation was inversely associated with pitting scars. Cutolo et al. [13] reported that capillary elongation was the most prevalent capillary finding (80\%) in rheumatoid arthritis, but presumably it has not been evaluated for its association with the SSc clinical findings yet.

None of the capillary components was associated with PAH and HRCT involvement in SSc patients in our study. Sato et al. [14] found no association between the number of capillary loops, number of giant capillary loops, and vascular deletion score with PAH and interstitial lung fibrosis, either. However, Hofstee et al. [15] 
found it lower in SSc patients with PAH than those without it. On the other hand, Riccieri et al. [16] found that the presence of avascular area was associated with $\mathrm{PAH}$ in SSc patients compared to those without it. Also, Ong et al. [17] stated that reduced capillary density was associated with PAH and there might be a pathogenic and etiologic relationship between them.

Regarding the HRCT involvement, Bredemeier et al. [18] found that higher avascular area score was associated with ground glass opacities in SSc (giving higher scores to more severe avascular areas), which we could not corroborate.

The capillary dilatation was not significantly different between those with PAH and without it both in our study and that of Hofstee et al. [15]. So, we had similar results in this regard.

Regarding capillary density, though, we could not find any significant relationship between lower capillary density and limitation of FTP range of motion of fingers, when analyzed in all patients.

Although capillary anomalies were not associated with any clinical finding, neoangiogenesis (a subdivision of capillary anomalies) was associated with pitting scars. In contrast, Sato et al. [14] found a lower number of capillary loops in SSc patients with finger pad lesion. Moreover, we found giant loops more prevalent in those with dysphagia, which might be a similar result to those of Sato's et al. in that they found a higher number of giant loops associated with esophageal dysmotility [14]. Also, we found avascular area to be associated with Gl problems, for which there are no similar studies in the literature for comparison. In our study, we did not use scores for capillaroscopy findings, so we did not have any data about the association of severity of clinical findings and capillaroscopy scoring.

Abnormal blood flow in our study was associated with a higher skin score $(>15)$ with a mean skin score of 22.2 only when analyzed in the late SSc subgroup, but not when we analyzed all patients and other SSc subtypes/subgroups.

Regarding the laboratory data, abnormal blood flow was found to be associated with higher CRP in the present study. No similar results were found in the literature. However, Sato et al. [14] found a higher vascular deletion score associated with Anti-Scl 70.

Neoangiogenesis, reduced capillary density, avascular area, and hemorrhage had a direct association with limitation of FTP range of motion of fingers. Neoangiogenesis was directly associated with pitting scars, too. Also, avascular area had a direct association with Gl problems, and giant loops had a direct association with dysphagia. Abnormal blood flow showed a direct relationship with positive CRP when studied in all patients.
So, these capillaroscopic findings are probably associated with greater disease severity and progression.

An important point inferred from this study was that some capillary changes had an inverse association with some clinical findings when studied in all patients.

For example, subtle change was inversely associated with limitation of FTP range of motion of fingers and GI problem. Capillary elongation was inversely associated with pitting scars, and capillary tortuosity was inversely associated with peripheral vascular problems (including Raynaud's phenomenon requiring vasodilators, pitting scars, digital tip ulceration, and digital gangrene) when we analyzed all patients. These findings might be considered as good prognostic factors when detected in capillaroscopy studies.

An advantage of this study is that only one rheumatologist studied the capillaries compared to multicenter studies. Also, the consideration of both SSc subtypes (DcSSC and LCSSc) and cross-sectional disease duration mentioned as subgroups (early and late SSc) simultaneously was another advantage. The limitations were the lower number of patients with diffuse scleroderma and the very low number of patients with pulmonary hypertension. It was found in previous studies that progression from the 'early' scleroderma pattern of capillaroscopy to 'active' and 'late' patterns can correlate with internal organ involvement in patients with scleroderma [19]; however, in our study we did not follow the capillaroscopy patterns and association of their changes with clinical manifestations, and this could be the goal of future studies.

\section{Conclusions}

We found no association between the patterns of capillaroscopy with SSc subtypes (DcSSc and LcSSc), but a significant association was revealed when they were studied in the SSc subtypes based on the duration of disease. We detected the 'early' scleroderma pattern to be more prevalent in early LCSSc, but not in early DcSSc, which could be compatible with slower course of the disease in them. Moreover, we found a direct association between some capillary components with more severe clinical findings. These capillary components were neoangiogenesis, reduced capillary density, avascular area, hemorrhage, avascular area, giant loops, and abnormal blood flow. Subtle change, capillary elongation, and capillary tortuosity had an inverse association with some clinical manifestations; they might be considered as good prognostic factors.

\section{Acknowledgements}

The present article was extracted from the thesis written by Dr. Mahyar Yousefipour Haghighi and finan- 
cially supported by Shiraz University of Medical Science Grant number: 91-01-01-4609. The authors would like to thank the Center for Development of Clinical Research of Namazee Hospital and Dr. Nasrin Shokrpour for editorial assistance.

The authors declare no conflict of interest.

\section{References}

1. Longo DL. Harrison's Online: Featuring the Complete Contents of Harrison's Principles of Internal Medicine. McGraw-Hill, New York 2012.

2. Brown GE, O'Leary PA. Skin capillaries in scleroderma. Arch Internal Med 1925; 36: 73-88.

3. Cutolo M, Sulli A, Secchi ME, et al. The contribution of capillaroscopy to the differential diagnosis of connective autoimmune diseases. Best Pract Res Clin Rheum 2007; 21: 10931108.

4. Avouac J, Fransen J, Walker U, et al. Preliminary criteria for the very early diagnosis of systemic sclerosis: results of a Delphi Consensus Study from EULAR Scleroderma Trials and Research Group. Ann Rheum Dis 2011; 70: 476-481.

5. Cutolo M, Sulli A, Pizzorni C, Accardo S. Nailfold videocapillaroscopy assessment of microvascular damage in systemic sclerosis. J Rheum 2000; 27: 155-160.

6. Ingegnoli F, Ardoino I, Boracchi P, Cutolo M. Nailfold capillaroscopy in systemic sclerosis: Data from the EULAR scleroderma trials and research (EUSTAR) database. Microvascular Res 2013; 89: 122-128.

7. Lambova S, Mueller-Ladner U. Capillaroscopic findings in systemic sclerosis - are they associated with disease duration and presence of digital ulcers? Discovery Med 2011; 12: 413-418.

8. Medsger Jr. TA, Steen VD. Classification, prognostic. In: Systemic sclerosis. Clements PJ, Furst DE (eds.). Lippincott Williams \& Wilkins, Baltimore 1996; 51-64.
9. Hoogen F, Khanna D, Fransen J, et al. 2013 Classification Criteria for Systemic Sclerosis: An American College of Rheumatology/European League Against Rheumatism Collaborative Initiative. Arthritis Rheum 2013; 65: 2737-2747.

10. LeRoy EC, Black C, Fleischmajer R, et al. Scleroderma (systemic sclerosis): classification, subsets and pathogenesis. J Rheum 1988; 15: 202.

11. Corrado A, Carpagnano GE, Gaudio A, et al. Nailfold capillaroscopic findings in systemic sclerosis related lung fibrosis and in idiopathic lung fibrosis. Joint Bone Spine 2010; 77: 570-574.

12. Cutolo M, Pizzorni C, Tuccio M, et al. Nailfold videocapillaroscopic patterns and serum autoantibodies in systemic sclerosis. Rheumatology 2004; 43: 719-726.

13. Cutolo M. Atlas of capillaroscopy in rheumatic diseases. Elsevier, Milan 2011.

14. Sato LT, Kayser C, Andrade L. Nailfold capillaroscopy abnormalities correlate with cutaneous and visceral involvement in systemic sclerosis patients. Acta Reumatologica Portuguesa 2008; 34: 219-227.

15. Hofstee H, Noordegraaf AV, Voskuyl A, et al. Nailfold capillary density is associated with the presence and severity of pulmonary arterial hypertension in systemic sclerosis. Ann Rheum Dis 2009; 68: 191-195.

16. Riccieri V, Vasile M, Iannace N, et al. Systemic sclerosis patients with and without pulmonary arterial hypertension: a nailfold capillaroscopy study. Rheumatology 2013; 52: 1525-1528.

17. Ong YY, Nikoloutsopoulos T, Bond CP, et al. Decreased nailfold capillary density in limited scleroderma with pulmonary hypertension. Asian Pac J Allergy Immunol 1998; 16: 81-86.

18. Bredemeier M, Xavier RM, Capobianco KG, et al. Nailfold capillary microscopy can suggest pulmonary disease activity in systemic sclerosis. J Rheum 2004; 31: 286-294.

19. Chojnowski MM, Felis-Giemza A, Olesińska M. Capillaroscopy - a role in modern rheumatology. Reumatologia 2016; 54 : 67-72. 